\title{
First-line single-agent chemotherapy for patients with recurrent or metastatic gastric cancer with poor performance status
}

\author{
JUN-EUL HWANG ${ }^{1}$, HA-NA KIM ${ }^{1}$, DAE-EUN KIM ${ }^{1}$, HYUN-JEONG SHIM ${ }^{1}$, WOO-KYUN BAE ${ }^{1}$, \\ EU-CHANG HWANG ${ }^{2}$, SANG-HEE $\mathrm{CHO}^{1}$ and IK-JOO $\mathrm{CHUNG}^{1}$ \\ ${ }^{1}$ Division of Hematology-Oncology, Department of Internal Medicine, and ${ }^{2}$ Department of Urology, \\ Chonnam National University Hwasun Hospital, Jeonnam, Republic of Korea
}

Received April 18, 2012; Accepted July 19, 2012

DOI: $10.3892 / \mathrm{etm} .2012 .644$

\begin{abstract}
Combination chemotherapy is a standard treatment approach in advanced gastric cancer. However, combination chemotherapy for advanced gastric cancer is often associated with severe treatment-related toxicities and most oncologists are reluctant to perform combination chemotherapy in patients with a poor clinical condition. We retrospectively investigated the efficacy and tolerability of single-agent chemotherapy in patients with recurrent or metastatic gastric cancer with poor performance status (PS). We reviewed advanced gastric adenocarcinoma patients who received first-line single-agent palliative chemotherapy due to poor PS between June 2006 and December 2010. A total of 125 patients with Eastern Cooperative Oncology Group (ECOG) PS 2-3, whose general condition did not allow combination chemotherapy, were enrolled. Four single agents were used: TS-1 $(n=63)$, paclitaxel $(n=42)$, irinotecan $(n=15)$ and capecitabine $(n=5)$. The median age was 66 years, with a range of 25-81 years. The percent response rate and rate of stable disease (SD) were 19.2 and $35.2 \%$, respectively, giving a disease control rate of $54.4 \%$. The median progression-free survival (PFS) was 3.9 months (95\% CI, 2.73-5.06). The median overall survival (OS) was 9.1 months (95\% CI, 7.70-10.56) with a 1-year survival rate of $31.2 \%$. Multivariate analysis demonstrated that the independent prognostic factors for OS were chemotherapy regimen (capecitabine) [reference: TS-1, hazard ratio (HR), 5.00; 95\% CI, 1.81-13.81; $\mathrm{P}=0.002]$, no second-line chemotherapy (HR, 2.3; 95\% CI, 1.48-3.57; $\mathrm{P}=0.001)$, bone metastasis (HR, 2.73; 95\% CI, 1.22-6.09; P=0.014), ECOG PS 3 (HR, 38.10; 95\% CI, 13.72-105.78; $\mathrm{P}=0.001)$, Glasgow prognostic score $(\mathrm{GPS}) \geq 1$ (HR, 1.88; 95\% CI, 1.24-2.85; P=0.003) and chemotherapy response $[\mathrm{SD}+$ progressive disease $(\mathrm{PD})+$ not evaluable $(\mathrm{NE})$;
\end{abstract}

Correspondence to: Professor Ik-Joo Chung, Division of Hematology-Oncology, Department of Internal Medicine, Chonnam National University Hwasun Hospital, 322 Seoyang-ro, Hwasun-eup, Hwasun-gun, Jeonnam 519-763, Republic of Korea

E-mail: ijchung@chonnam.ac.kr

Key words: chemotherapy, stomach neoplasms, recurrence, metastasis
HR, 2.37; 95\% CI, 1.39-4.05; $\mathrm{P}=0.002)$ ]. First-line singleagent palliative chemotherapy demonstrated a relatively good clinical efficacy for recurrent or metastatic gastric cancer patients with poor PS.

\section{Introduction}

Recurrent or metastatic gastric cancer has a poor prognosis but chemotherapy improves survival and possibly provides significant palliation of symptoms. While newer agents for advanced gastric cancer have been developed in recent years, including TS-1, capecitabine, taxanes, oxaliplatin and irinotecan, the prognosis of unresectable, recurrent or metastatic gastric cancer patients remains extremely poor, with a survival of only 6-13 months (1-3).

Eastern Cooperative Oncology Group performance status (ECOG PS) is the most important parameter for predicting response to chemotherapy and survival, and is associated with a number of factors, including age, comorbidity and nutritional status $(4,5)$. ECOG PS 2 had a significant negative impact on survival in a number of other studies. PS classifications of $0-1$ and 2-3 are generally used to stratify patients in phase III trials on advanced gastric cancer due to their well-known impact on survival (6-8).

In recurrent or metastatic gastric cancer, patients are likely to suffer from poor general condition due to anorexia and weight loss, often as a consequence of peritoneal carcinomatosis. These patients have been excluded from clinical trials and chemotherapeutic options are limited $(9,10)$. Most oncologists select cisplatin-based combination chemotherapy for recurrent or metastatic gastric cancer patients with good PS in the first-line setting. Multidrug combination chemotherapy regimens have generally provided higher response rates, but also more substantial toxicities (11) and do not significantly improve overall survival (OS). These toxicities reduce their value as a palliative treatment and are of particular concern in patients whose PS is compromised (12). Selecting a chemotherapy regimen for an individual patient is a common clinical situation and factors such as the extent of disease and potential toxicities must be considered, especially for patients with poor PS.

The single-agent chemotherapy drug TS-1 (Taiho Pharmaceutical Company, Tokyo, Japan) has demonstrated 
good clinical efficacy for advanced gastric cancer (13). Therefore, in the present study, we retrospectively evaluated patients with unresectable, recurrent or metastatic gastric cancer who received first-line single-agent palliative chemotherapy due to poor PS (ECOG PS 2-3).

\section{Patients and methods}

Patients. We evaluated patients with advanced gastric cancer who had received first-line single-agent palliative chemotherapy between June 2006 and December 2010 at Chonnam National University Hwasun Hospital (Gwangju, Korea). Patients were staged using a combination of endoscopy, computed tomography (CT) scans of the chest and abdomen and positron emission tomography or bone scans when clinically indicated.

The criteria for case inclusion were as follows: i) histologically confirmed gastric adenocarcinoma; ii) no prior chemotherapy or radiotherapy, with the exception of adjuvant treatment; iii) presence of metastatic disease; and iv) availability of clinical data at the initiation of therapy and during follow-up. Of the 201 patients screened, 125 fulfilled the inclusion criteria and were enrolled in this retrospective analysis. We collected follow-up patient data from the cancer registry. All data were prospectively recorded and only the survival data were updated at the time of analyses.

ECOG PS was evaluated according to the ECOG criteria. The clinical tumor response was assessed radiologically by CT scanning after every 2 or 3 courses of chemotherapy according to the Response Evaluation Criteria in Solid Tumors (RECIST version 1.0) (14).

The chemotherapy regimens included TS-1 $(n=63)$, paclitaxel $(n=42)$, irinotecan $(n=15)$ and capecitabine $(n=5)$. TS-1 was given at a dose of $80 \mathrm{mg} / \mathrm{m}^{2} /$ day for 4 weeks, followed by a 2 -week rest (13). Paclitaxel was administered at a dose of $80 \mathrm{mg} / \mathrm{m}^{2}$ by intravenous infusion, weekly for 3 weeks of a 4 week cycle (days 1, 8 and 15 of each cycle) (15). Irinotecan was administered at a dose of $150 \mathrm{mg} / \mathrm{m}^{2}$ by intravenous infusion every 2 weeks (days 1 and 15 of each cycle) $(16,17)$. Capecitabine was administered at a dose of $1,250 \mathrm{mg} / \mathrm{m}^{2} /$ day for 2 weeks, followed by a 1-week rest (18).

Treatment was continued until the occurrence of disease progression, lack of clinical benefit, unacceptable toxicity or patient refusal. Toxicities were graded according to the National Cancer Institute Common Terminology Criteria for Adverse Events version 3.0 (CTCAE). The dose of the subsequent cycles was adjusted according to the toxic effects that developed during the preceding cycle.

This study was approved by the Institutional Review Board of Chonnam National University Medical School Research Institution.

Statistics. Kaplan-Meier analysis was applied to assess factors affecting OS and progression-free survival (PFS) and the significance of differences between survival curves was determined by the log-rank test. OS was defined as the period from the date of the first course of chemotherapy to the date of mortality from any cause. PFS was defined as the period from the date of the first course of chemotherapy to the date of disease progression or mortality, whichever occurred first.
Table I. Patient characteristics.

\begin{tabular}{|c|c|}
\hline Characteristics & $\begin{array}{l}\text { No. of patients } \\
\qquad(\mathrm{n}=125)\end{array}$ \\
\hline \multicolumn{2}{|l|}{ Age (years) } \\
\hline Median (range) & $66(25-81)$ \\
\hline \multicolumn{2}{|l|}{ Gender } \\
\hline Male/female & $90 / 35$ \\
\hline \multicolumn{2}{|l|}{ ECOG PS } \\
\hline $2 / 3$ & $110 / 15$ \\
\hline \multicolumn{2}{|l|}{ Histological grade } \\
\hline Adenocarcinoma, WD & 10 \\
\hline Adenocarcinoma, MD & 38 \\
\hline Adenocarcinoma, PD & 57 \\
\hline Signet ring cell carcinoma & 12 \\
\hline Unknown & 8 \\
\hline \multicolumn{2}{|l|}{ Lauren classification } \\
\hline Diffuse & 40 \\
\hline Intestinal & 63 \\
\hline Mixed & 14 \\
\hline Unknown & 8 \\
\hline Previous gastrectomy (yes/no) & $61 / 64$ \\
\hline Second-line chemotherapy (yes/no) & $65 / 60$ \\
\hline \multicolumn{2}{|l|}{ Metastatic site } \\
\hline Liver & 42 \\
\hline Peritoneum & 48 \\
\hline Bone & 7 \\
\hline \multicolumn{2}{|l|}{ Albumin (g/dl) } \\
\hline$<3.5$ & 38 \\
\hline$\geq 3.5$ & 87 \\
\hline \multicolumn{2}{|l|}{ CRP (mg/dl) } \\
\hline$\leq 1.0$ & 57 \\
\hline$>1.0$ & 68 \\
\hline \multicolumn{2}{|l|}{ GPS } \\
\hline $0 / 1 / 2$ & $50 / 44 / 31$ \\
\hline
\end{tabular}

ECOG PS, Eastern Cooperative Oncology Group performance status; WD, well-differentiated; MD, moderately differentiated; PD, poorly differentiated; CRP, C-reactive protein; GPS, Glasgow prognostic score.

If neither event had occurred at the time of the last record, the patient was censored at that time. The factors included in univariate survival analysis were age, gender, histological grade, Lauren classification, previous gastrectomy, chemotherapy regimen, chemotherapy response, second-line chemotherapy, liver metastasis, peritoneal metastasis, bone metastasis, albumin, C-reactive protein (CRP) concentration and Glasgow prognostic score (GPS). GPS is known to be associated with prognosis in gastric cancer. Patients with both an elevated CRP concentration $(>1.0 \mathrm{mg} / \mathrm{dl})$ and hypoalbuminemia $(<3.5 \mathrm{mg} / \mathrm{dl})$ were assigned a GPS of 2 . Patients in 
Table II. Toxicities.

No. of patients $(n=125)$

\begin{tabular}{|c|c|c|c|c|c|c|}
\hline \multirow[b]{2}{*}{ Toxicity } & \multicolumn{4}{|c|}{ No. of patients $(n=125)$} & \multirow{2}{*}{$\begin{array}{c}\text { All grades } \\
(\%)\end{array}$} & \multirow{2}{*}{$\begin{array}{c}\text { Grade } 3 / 4 \\
(\%)\end{array}$} \\
\hline & Grade 1 & Grade 2 & Grade 3 & Grade 4 & & \\
\hline \multicolumn{7}{|l|}{ Hematological } \\
\hline Leukopenia & 34 & 14 & 14 & 9 & 57 & 18 \\
\hline Neutropenia (febrile) & 33 & 13 & $11(3)$ & $6(1)$ & 50 & 14 \\
\hline Anemia & 45 & 47 & 6 & 4 & 82 & 8 \\
\hline Thrombocytopenia & 13 & 9 & 3 & 0 & 20 & 2 \\
\hline \multicolumn{7}{|l|}{ Non-hematological } \\
\hline Asthenia/anorexia & 9 & 4 & 13 & 0 & 21 & 10 \\
\hline Nausea/vomiting & 8 & 5 & 10 & 0 & 18 & 8 \\
\hline Mucositis & 13 & 8 & 0 & 0 & 17 & 0 \\
\hline Diarrhea & 16 & 5 & 8 & 0 & 23 & 6 \\
\hline Peripheral neuropathy & 9 & 11 & 1 & 0 & 17 & 1 \\
\hline Hand-foot syndrome & 3 & 3 & 1 & 0 & 6 & 1 \\
\hline
\end{tabular}

Table III. Chemotherapy regimens $(\mathrm{n}=125)$.

\begin{tabular}{lcc}
\hline Regimen & No. of patients & $\%$ \\
\hline TS-1 & 63 & 50.4 \\
Paclitaxel & 42 & 33.6 \\
Irinotecan & 15 & 12 \\
Capecitabine & 5 & 4 \\
\hline
\end{tabular}

whom only 1 of these biochemical abnormalities was present were assigned a GPS of 1 and patients with normal CRP and albumin levels were assigned a score of 0 (19). Multivariate regression analysis using the Cox proportional hazards regression model (the stepwise forward procedure), was performed to achieve an adjusted hazard ratio (HR) to determine prognostic factors for OS and PFS. A two-tailed $\mathrm{P}<0.05$ was considered to indicate a statistically significant result for all analyses. The SPSS software package, version 17.0 (SPSS, Inc., Chicago, IL, USA) was used for statistical analysis.

\section{Results}

Patient characteristics. The baseline characteristics of the 125 patients are listed in Table I. The median follow-up time was 9.1 months, with a range of 1.7-34.2 months. The median age of the patients was 66 years, with a range of 25-81 years. A total of 90 patients (72\%) were male and 110 patients $(88 \%)$ had a ECOG PS of 2. A total of 97 patients $(77.6 \%)$ had measurable metastatic lesions. A total of 42 patients $(33.6 \%)$ had liver metastases and 48 patients (38.4\%) and 7 patients (5.6\%) had peritoneal and bone metastases, respectively. A total of 61 patients $(48.8 \%)$ had undergone gastrectomy prior to palliative chemotherapy. A total of 68 patients $(54.4 \%)$ had an elevated CRP level ( $>1 \mathrm{mg} / \mathrm{dl})$ and 38 patients $(30.4 \%)$ had hypoalbuminemia $(<3.5 \mathrm{mg} / \mathrm{dl})$. A total of 65 patients $(52 \%)$ received second-line chemotherapy.
Table IV. Response to chemotherapy ( $n=125)$.

\begin{tabular}{lcr}
\hline Response & No. of patients & $\%$ \\
\hline Complete response & 3 & 2.4 \\
Partial response & 21 & 16.8 \\
Stable disease & 44 & 35.2 \\
Progressive disease & 51 & 40.8 \\
Not evaluable & 6 & 4.8 \\
\hline
\end{tabular}

Toxicities. A total of 482 chemotherapy cycles were administered. The patients received a median of 3 (range, 1-12) cycles. The most common reason for treatment discontinuation was disease progression. Toxicity was assessed in all patients. The chemotherapy toxicities during the total treatment courses are summarized in Table II. Hematological toxicities were relatively common. The incidence of grade 3 or 4 leukopenia and neutropenia were 18 and 14\%, respectively. Four patients developed febrile neutropenia. Three of these patients recovered with systemic antibiotics and granulocyte colony-stimulating factor, while 1 patient succumbed to neutropenic sepsis after receiving weekly paclitaxel. Anemia was the most common adverse event, since 79 of the 125 patients $(63.2 \%)$ had anemia before the initiation of the chemotherapy. Three patients $(2 \%)$ had grade 3 thrombocytopenia; 2 in the paclitaxel group and 1 in the irinotecan group. Non-hematological toxicities consisted of asthenia/anorexia (21\%), nausea/vomiting (18\%), mucositis (17\%), diarrhea (23\%) and hand-foot syndrome (6\%). Peripheral neuropathy developed in 21 patients $(17 \%)$ but was mild with the exception of 1 patient. All patients with peripheral neuropathy were in the paclitaxel group.

Response and survival. The most commonly used first-line single-agent chemotherapy regimen was TS-1 $(n=63,50.4 \%)$. The specific chemotherapy regimens are shown in Table III. Of 
Table V. Univariate analysis of clinical factors for overall survival $(n=125)$.

$\overline{\text { mOS months }(95 \% \mathrm{CI}) \quad \mathrm{P} \text {-value }}$

Age (years)
$<66$
$\geq 66$

Gender

Male

Female

$8.7(6.29-11.1)$

$9.5(7.64-11.49)$

0.506

$8.7(7.09-10.30)$

$10.8(8.5-13.1)$

Type

Diffuse
Intestinal
Mixed
Unknown

$8.9(7.89-10.04)$

$10.1(7.61-12.58)$

$8.3(0.00-17.10)$

$7.0(2.98-11.01)$

Previous gastrectomy

Yes

No

$9.5(7.85-11.27)$

$8.4(5.78-11.01)$

Liver metastasis

Yes

No

$7.2(4.34-10.05)$

9.5 (8.21-10.92)

Peritoneal metastasis

Yes

No

$8.9(7.27-10.59)$

9.5 (7.56-11.57)

Chemotherapy regimen

TS-1

Paclitaxel

$9.5(7.27-11.86)$

$9.0(6.91-11.15)$

$8.2(5.57-10.96)$

$6.3(0.00-13.45)$

Capecitabine

Second-line chemotherapy

Yes

No

$11.2(10.51-12.02)$

$6.6(5.57-7.76)$

Bone metastasis

Yes

No

$7.3(7.04-7.55)$

$9.5(8.08-11.04)$

0.390

Chemotherapy response
$\mathrm{CR}+\mathrm{PR}$
$\mathrm{SD}+\mathrm{PD}$
$\mathrm{NE}$

$14.8(10.13-19.60)$

$8.7(7.30-10.09)$

$3.0(0.00-7.66)$

Histological grade

Adenocarcinoma, WD

Adenocarcinoma, MD

Adenocarcinoma, PD

Signet ring cell carcinoma

Unknown

\section{ECOG PS}

2

3

$10.3(8.91-11.75)$

$3.0(2.36-3.63)$

Albumin (g/dl)

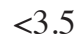

$\geq 3.5$

0.226

0.355

0.567

0.830

0.001

0.379

$7.2(4.77-9.62)$

$10.7(8.19-13.33)$

$8.9(7.06-10.86)$

$13.5(5.89-21.17)$

$7.0(2.98-11.01)$

0.001
Table V. Continued.

mOS months $(95 \% \mathrm{CI}) \quad \mathrm{P}$-value

\begin{tabular}{lcc}
\hline $\mathrm{CRP}(\mathrm{mg} / \mathrm{dl})$ & & \\
$\leq 1.0$ & $11.8(8.26-15.33)$ & 0.001 \\
$>1.0$ & $7.1(6.15-8.11)$ & \\
GPS & & \\
0 & $11.8(7.99-15.60)$ & 0.001 \\
$\geq 1$ & $7.4(6.39-8.40)$ & \\
\hline
\end{tabular}

mOS, median overall survival; $\mathrm{CR}$, complete response; $\mathrm{PR}$, partial response; $\mathrm{SD}$, stable disease; $\mathrm{PD}$, progressive disease; $\mathrm{NE}$, not evaluable; WD, well-differentiated; MD, moderately differentiated; PD, poorly differentiated; ECOG PS, Eastern Cooperative Oncology Group performance status; CRP, C-reactive protein; GPS, Glasgow prognostic score.

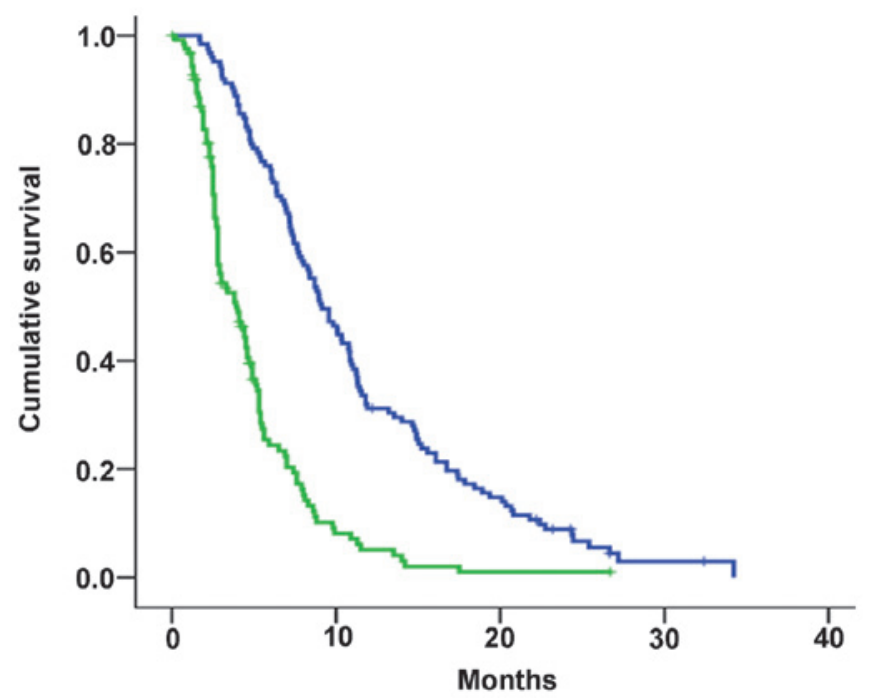

Figure 1. OS and PFS curve for all patients. The median OS and PFS were 9.1 (95\% CI, 7.70-10.56) and 3.9 months (95\% CI, 2.73-5.06), respectively, with a 1-year survival rate of $31.2 \%$ (blue line, OS; green line, PFS. OS, overall survival; PFS, progression-free survival.

a total of 125 patients, 6 could not be evaluated for responses because of early discontinuation of therapy. A complete response (CR) to chemotherapy was achieved in 3 patients $(2.4 \%)$ and a partial response (PR) in 21 patients $(16.8 \%)$. The percentage of stable disease (SD) was $35.2 \%$, giving an overall response rate of $19.2 \%$ and a disease control rate of $54.4 \%$. Objective tumor response to each regimen was $19 \%$ for TS-1, 21.4\% for paclitaxel, $13.3 \%$ for irinotecan and $20 \%$ for capecitabine. The chemotherapy results are shown in Table IV.

The median PFS was 3.9 months (95\% CI, 2.73-5.06). The median OS was 9.1 months (95\% CI, 7.70-10.56) with a 1-year survival rate of $31.2 \%$ (Fig. 1). In univariate analysis, the median OS was significantly shorter for patients with the following clinical factors: no second-line chemotherapy, response of stable and progressive disease (PD) to chemotherapy, ECOG PS 3, CRP $>1.0 \mathrm{mg} / \mathrm{dl}$ and GPS $\geq 1$ (Table V). 
Table VI. Univariate analysis of clinical factors for progression-free survival $(n=125)$.

mPFS months (95\% CI) P-value

Age (years)
$<66$
$\geq 66$

$3.2(2.23-4.30)$

Gender

Male

Female

$4.3(3.38-5.49)$

$3.9(2.66-5.27)$

$3.7(2.00-5.52)$

Type

$\begin{array}{ll}\text { Diffuse } & 4.1(1.90-6.29) \\ \text { Intestinal } & 3.9(2.73-5.20) \\ \text { Mixed } & 2.9(0.00-5.86) \\ \text { Unknown } & 2.8(2.06-3.60)\end{array}$

Previous gastrectomy

Yes

No

$3.9(2.34-5.59)$

$3.8(2.11-5.61)$

Liver metastasis

Yes

No

$2.8(2.59-3.07)$

$4.3(3.59-5.13)$

Peritoneal metastasis

Yes

No

$2.8(1.48-4.11)$

$4.3(3.30-5.42)$

Chemotherapy regimen

TS-1

Paclitaxel

Irinotecan

Capecitabine

$4.3(3.58-5.15)$

$3.7(2.93-4.60)$

$2.8(1.82-3.84)$

$2.0(1.56-2.56)$

Second-line chemotherapy

Yes

No

$3.9(2.44-5.48)$

$3.7(2.16-5.36)$

Bone metastasis

Yes

No

$4.3(2.09-6.63)$

$3.7(2.53-5.00)$

Chemotherapy response

CR+PR
SD+PD
NE

Histological grade

Adenocarcinoma, WD

Adenocarcinoma, MD

Adenocarcinoma, PD

Signet ring cell carcinoma

Unknown

$7.0(5.05-9.01)$

$2.8(2.58-3.08)$

$2.0(1.71-2.41)$

$2.7(2.19-3.33)$

$4.1(2.73-5.59)$

$4.1(2.25-5.94)$

$2.7(2.45-3.07)$

$2.8(2.06-3.60)$

\section{ECOG PS}

2

$4.4(3.79-5.14)$

$1.9(1.35-2.44)$

0.001

3

Albumin (g/dl)

$<3.5$

$\geq 3.5$

0.704

0.570

0.377

0.133

0.483
Table VI. Continued.

mPFS months $(95 \% \mathrm{CI}) \quad \mathrm{P}$-value

\begin{tabular}{lll}
\hline CRP $(\mathrm{mg} / \mathrm{dl})$ & & \\
$\leq 1.0$ & $4.4(3.52-5.41)$ & 0.064 \\
$>1.0$ & $3.0(1.73-4.26)$ & \\
GPS & & \\
0 & $4.6(3.50-5.69)$ & 0.006 \\
$\geq 1$ & $2.8(2.29-3.37)$ & \\
\hline
\end{tabular}

mPFS, median progression-free survival; CR, complete response; $P R$, partial response; $\mathrm{SD}$, stable disease; $\mathrm{PD}$, progressive disease; $\mathrm{NE}$, not evaluable; WD, well-differentiated; MD, moderately differentiated; PD, poorly differentiated; ECOG PS, Eastern Cooperative Oncology Group performance status; CRP, C-reactive protein; GPS, Glasgow prognostic score.

Univariate analysis also demonstrated that 3 clinical factors were significantly associated with a shorter PFS; these factors included response of SD and PD to chemotherapy, ECOG PS 3 and GPS $\geq 1$ (Table VI).

Multivariate regression analysis using the Cox proportional hazards regression model (the stepwise forward procedure) was performed. The result of the analysis identified the independent poor prognostic factors for OS and PFS (Table VII). The independent poor prognostic factors for OS were chemotherapy regimen (capecitabine; reference: TS-1, HR, 5.00; 95\% CI, 1.81-13.81; P=0.002), no secondline chemotherapy (HR, 2.30; 95\% CI, 1.48-3.57; $\mathrm{P}=0.001)$, bone metastasis (HR, 2.73; 95\% CI, 1.22-6.09; $\mathrm{P}=0.014$ ), ECOG PS 3 (HR, 38.10; 95\% CI, 13.72-105.78; $\mathrm{P}=0.001$ ), GPS $\geq 1$ (HR, 1.88; 95\% CI, 1.24-2.85; $\mathrm{P}=0.003$ ) and chemotherapy response $[\mathrm{SD}+\mathrm{PD}+$ not evaluable $(\mathrm{NE}) ; \mathrm{HR}, 2.37$; 95\% CI, 1.39-4.05; $\mathrm{P}=0.002)]$. The independent prognostic factors for PFS were chemotherapy regimen (capecitabine; reference: TS-1; HR, 4.20; 95\% CI, 1.64-10.85; $\mathrm{P}=0.003$ ), ECOG PS 3 (HR, 5.86; 95\% CI, 3.07-11.20; P=0.001) and chemotherapy response (SD + PD + NE; HR, 2.41; 95\% CI, 1.44-4.04; $\mathrm{P}=0.001)$. After first-line failure, second-line chemotherapy was administered in 65 patients (52\%). OS was longer in the group able to receive second-line chemotherapy (11.2 vs. 6.6 months, $\mathrm{P}=0.004)$.

\section{Discussion}

Currently, fluoropyrimidine and cisplatin combination chemotherapy is accepted as a standard regimen by numerous oncologists. However, cisplatin is associated with significant toxicity and usually requires careful clinical monitoring and supportive care, including intensive intravenous hydration. Patients with poor PS usually have several comorbidities and are also more vulnerable to the toxicity of chemotherapy (11). Therefore, we evaluated the efficacy of single-agent chemotherapy with TS-1, paclitaxel, capecitabine and irinotecan. TS-1, paclitaxel and capecitabine are the counterparts of a cisplatin-based combination chemotherapy for advanced gastric cancer (11). 
Table VII. Overall survival and progression-free survival in advanced gastric cancer patients with poor performance status receiving single-agent chemotherapy (multivariate analysis).

\begin{tabular}{|c|c|c|}
\hline Factors & Hazard ratio $(95 \% \mathrm{CI})$ & P-value \\
\hline \multicolumn{3}{|l|}{ Overall survival } \\
\hline Chemotherapy regimen & & 0.016 \\
\hline Paclitaxel & $1.40(0.90-2.18)$ & 0.129 \\
\hline Irinotecan & $1.41(0.77-2.58)$ & 0.255 \\
\hline Capecitabine & $5.00(1.81-13.81)$ & 0.002 \\
\hline No second-line chemotherapy & $2.30(1.48-3.57)$ & 0.001 \\
\hline Bone metastasis & $2.73(1.22-6.09)$ & 0.014 \\
\hline ECOG PS 3 & $38.10(13.72-105.78)$ & 0.001 \\
\hline GPS $\geq 1$ & $1.88(1.24-2.85)$ & 0.003 \\
\hline \multicolumn{3}{|l|}{ Chemotherapy response } \\
\hline $\mathrm{SD}+\mathrm{PD}+\mathrm{NE}$ & $2.37(1.39-4.05)$ & 0.002 \\
\hline \multicolumn{3}{|l|}{ Progression-free survival } \\
\hline Chemotherapy regimen & & 0.025 \\
\hline Paclitaxel & $1.31(0.85-2.03)$ & 0.213 \\
\hline Irinotecan & $1.12(0.58-2.14)$ & 0.728 \\
\hline Capecitabine & $4.20(1.64-10.85)$ & 0.003 \\
\hline ECOG PS 3 & $5.86(3.07-11.20)$ & 0.001 \\
\hline Chemotherapy response & & 0.003 \\
\hline $\mathrm{SD}+\mathrm{PD}+\mathrm{NE}$ & $2.41(1.44-4.04)$ & 0.001 \\
\hline
\end{tabular}

ECOG PS, Eastern Cooperative Oncology Group performance status; GPS, Glasgow prognostic score; SD, stable disease; PD, progressive disease; NE, not evaluable. The reference value is TS-1 in chemotherapy regimen.

PS is an indicator of a patient's global ability and it correlates with survival time. Preoperative ECOG PS 2-3 in incurable gastric cancer patients is associated with a 1-year survival rate of $17 \%$, compared with $43 \%$ for ECOG PS $0-1$ patients (20). A phase II study of TS-1 demonstrated modest activity of TS-1 in gastric cancer patients with poor PS. The response rate was $12 \%$, with a 1-year survival rate of $29 \%$. This study included a more heterogeneous group of patients who received TS-1 as first- or second-line treatment (12). In a prospective trial of TS-1 plus cisplatin versus TS-1 alone (SPIRITS trial), the median PFS and OS of the TS-1 group were 4.0 and 11.0 months, respectively, with a 1-year survival rate of $46.7 \%$ and almost all patients $(97 \%)$ in this trial had a ECOG PS 0-1 (2). Our study demonstrated median PFS and OS of 3.9 and 9.1 months, respectively, with a 1-year survival rate of $31.2 \%$ and a response rate of $19.2 \%$. This suggests a relatively good efficacy of single-agent chemotherapy in gastric cancer patients in this poor PS population.

In the present study, there were no statistically significant differences between the regimens used, with the exception of capecitabine. TS-1 is an oral anticancer drug that combines tegafur, a prodrug of fluouracil, with 5-chloro2,4-dihydropyrimidine (CDHP) and potassium oxonate (21). TS-1 is widely used as first-line treatment for advanced gastric cancer in Asian countries. Phase II studies of TS-1 have noted responses of $44-54 \%$ in patients with advanced gastric cancer, but TS-1 has lower responses of $13-33 \%$ in this type of cancer when combined with poor clinical condition, including advanced age and poor PS $(12,22,23)$. Weekly paclitaxel and irinotecan regimens are usually used as second-line treatment for advanced gastric cancer with a response rate of $14-21 \%(24,25)$. Although direct comparison is difficult, our results are comparable to others for single-agent chemotherapy regimens in poor performers or pretreated patients. Capecitabine is mostly used as a combination regimen and when used as a single-agent, it demonstrates a response rate of $26-34 \%$ (18). In our study, capecitabine demonstrated a relatively low efficacy in PFS and OS, but we propose that this was due to the small number of patients in the capecitabine group.

We also evaluated the clinical factors associated with poor survival in this subgroup of recurrent or metastatic gastric cancer. No second-line chemotherapy, bone metastasis, ECOG PS 3, responses to chemotherapy $(\mathrm{SD}+\mathrm{PD}+\mathrm{NE})$ and GPS $\geq 1$ were significant survival predictors. It has been reported that GPS is associated with prognosis in various types of cancer, including non-small cell lung, gastric, colorectal, pancreatic and breast cancers. Previously, we reported that GPS is a useful predictor of survival in patients with recurrent or metastatic gastric cancer receiving palliative chemotherapy (19). GPS was a useful prognostic indicator in this subgroup of recurrent or metastatic gastric cancer. 
In Korea, it has been common practice for patients who fail first-line palliative chemotherapy to receive second-line chemotherapy and Kang et al reported that salvage chemotherapy (second- or third-line chemotherapy) in advanced gastric cancer resulted in significant prolongation of survival when compared with best supportive care (17). Second-line chemotherapy was also a significant survival predictor in our study of recurrent or metastatic gastric cancer patients with poor performance status.

In conclusion, first-line single-agent palliative chemotherapy is tolerated and has a relatively good clinical efficacy for the treatment of recurrent or metastatic gastric cancer patients with poor PS.

\section{References}

1. Bang YJ, Van Cutsem E, Feyereislova A, et al: Trastuzumab in combination with chemotherapy versus chemotherapy alone for treatment of HER2-positive advanced gastric or gastrooesophageal junction cancer (ToGA): a phase 3, open-label, randomised controlled trial. Lancet 376: 687-697, 2010.

2. Koizumi W, Narahara H, Hara T, et al: S-1 plus cisplatin versus S-1 alone for first-line treatment of advanced gastric cancer (SPIRITS trial): a phase III trial. Lancet Oncol 9: 215-221, 2008.

3. Vanhoefer U, Rougier P, Wilke H, et al: Final results of a randomized phase III trial of sequential high-dose methotrexate, fluorouracil, and doxorubicin versus etoposide, leucovorin and fluorouracil versus infusional fluorouracil and cisplatin in advanced gastric cancer: A trial of the European Organization for Research and Treatment of Cancer Gastrointestinal Tract Cancer Cooperative Group. J Clin Oncol 18: 2648-2657, 2000.

4. Lavin PT, Bruckner HW and Plaxe SC: Studies in prognostic factors relating to chemotherapy for advanced gastric cancer. Cancer 50: 2016-2023, 1982.

5. Wilson D, Hiller L and Geh JI: Review of second-line chemotherapy for advanced gastric adenocarcinoma. Clin Oncol (R Coll Radiol) 17: 81-90, 2005.

6. Boku N, Yamamoto S, Fukuda H, et al: Fluorouracil versus combination of irinotecan plus cisplatin versus S-1 in metastatic gastric cancer: a randomised phase 3 study. Lancet Oncol 10 1063-1069, 2009.

7. Lee J, Lim T, Uhm JE, et al: Prognostic model to predict survival following first-line chemotherapy in patients with metastatic gastric adenocarcinoma. Ann Oncol 18: 886-891, 2007.

8. Chau I, Norman AR, Cunningham D, Waters JS, Oates J and Ross PJ: Multivariate prognostic factor analysis in locally advanced and metastatic esophago-gastric cancer-pooled analysis from three multicenter, randomized, controlled trials using individual patient data. J Clin Oncol 22: 2395-2403, 2004.

9. Cunningham D, Hole D, Taggart DJ, Soukop M, Carter DC and McArdle CS: Evaluation of the prognostic factors in gastric cancer: the effect of chemotherapy on survival. Br J Surg 74: 715-720, 1987.
10. Hsu CH, Yeh KH, Chen LT, et al: Weekly 24-hour infusion of high-dose 5-fluorouracil and leucovorin in the treatment of advanced gastric cancers. An effective and low-toxic regimen for patients with poor general condition. Oncology 54: 275-280, 1997.

11. Lim do H, Park SH, Park KW, et al: Retrospective analyses of cisplatin-based doublet combination chemotherapy in patients with advanced gastric cancer. BMC Cancer 10: 583, 2010.

12. Jeung HC, Rha SY, Shin SJ, Ahn JB, Noh SH, Roh JK and Chung HC: A phase II study of S-1 monotherapy administered for 2 weeks of a 3-week cycle in advanced gastric cancer patients with poor performance status. Br J Cancer 97: 4584-4563, 2007.

13. Hosokawa A, Sugiyama T, Ohtsu A, et al: Long-term outcomes of patients with metastatic gastric cancer after initial S-1 monotherapy. J Gastroenterol 42: 533-538, 2007.

14. Therasse P, Arbuck SG, Eisenhauer EA, et al: New guidelines to evaluate the response to treatment in solid tumors. European Organization for Research and Treatment of Cancer, National Cancer Institute of the United States, National Cancer Institute of Canada. J Natl Cancer Inst 92: 205-216, 2000.

15. Egawa T, Kubota T, Nagashima A, et al: Usefulness of weekly administration of paclitaxel for advanced or recurrent gastric cancer. Gan To Kagaku Ryoho 31: 877-881, 2004 (In Japanese).

16. Futatsuki K, Wakui A, Nakao I, et al: Late phase II study of irinotecan hydrochloride (CPT-11) in advanced gastric cancer. CPT-11 Gastrointestinal Cancer Study Group. Gan To Kagaku Ryoho 21: 1033-1038, 1994 (In Japanese).

17. Kang JH, Lee SI, Lim DH, et al: Salvage chemotherapy for pretreated gastric cancer: a randomized phase III trial comparing chemotherapy plus best supportive care with best supportive care alone. J Clin Oncol 30: 1513-1518, 2012.

18. Bang YJ. Capecitabine in gastric cancer. Expert Rev Anticancer Ther 11: 1791-1806, 2011.

19. Hwang JE, Kim HN, Kim DE, et al: Prognostic significance of a systemic inflammatory response in patients receiving first-line palliative chemotherapy for recurred or metastatic gastric cancer. BMC Cancer 11: 489, 2011

20. Maehara Y, Sugimachi K, Ogawa M, Kakegawa T, Shimazu H and Tomita M: Influence of preoperative performance status on survival time of patients with advanced gastric cancer following noncurative resection. Anticancer Res 13: 201-203, 1993.

21. Shirasaka T, Shimamato Y, Ohshimo H, Yamaguchi M, Kato T, Yonekura K and Fukushima M: Development of a novel form of an oral 5-fluorouracil derivative (S-1) directed to the potentiation of the tumor selective cytotoxicity of 5-fluorouracil by two biochemical modulators. Anticancer Drugs 7: 548-557, 1996.

22. Koizumi W, Akiya T, Sato A, et al: Phase II study of S-1 as first-line treatment for elderly patients over 75 years of age with advanced gastric cancer: the Tokyo Cooperative Oncology Group study. Cancer Chemother Pharmacol 65: 1093-1099, 2010.

23. Tsushima T, Hironaka S, Boku N, et al: Safety and efficacy of S-1 monotherapy in elderly patients with advanced gastric cancer. Gastric Cancer 13: 245-250, 2010.

24. Pasini F, Fraccon AP and DE Manzoni G. The role of chemotherapy in metastatic gastric cancer. Anticancer Res 31: 3543-3554, 2011.

25. Ando T, Hosokawa A, Kajiura S, et al: Efficacy of weekly paclitaxel in patients with advanced gastric cancer: refractory to docetaxel-based chemotherapy. Gastric Cancer Jan 18, 2012 (Epub ahead of print). 